\title{
Experimental Study on Relationship between Free Load Swelling Rate and Water Content of Medium Expansive Soil
}

\author{
Cheng Song, ${ }^{1}$ Ligong Yang $\mathbb{D}^{2}{ }^{2}$ Wei Xia, ${ }^{1}$ Wendong $\mathrm{Ji}^{2}{ }^{2}$ and Yuting Zhang ${ }^{2}$ \\ ${ }^{1}$ Anhui Provincial Group Limited for Yangtze-to-Huaihe Water Diversion, Hefei 230011, China \\ ${ }^{2}$ Tianjin Research Institute for water transport engineering, M.O.T., Tianjin 300456, China \\ Correspondence should be addressed to Ligong Yang; 534332303@qq.com
}

Received 28 September 2020; Revised 31 December 2020; Accepted 20 March 2021; Published 26 March 2021

Academic Editor: Francesco Colangelo

Copyright (c) 2021 Cheng Song et al. This is an open access article distributed under the Creative Commons Attribution License, which permits unrestricted use, distribution, and reproduction in any medium, provided the original work is properly cited.

Expansive soil has the property of water swelling, which is related not only to the hydrophilic mineral composition of soil particles and the microstructure of soil, but also to the initial moisture content, dry density, and overburden condition of soil. Based on the typical expansive soil in a certain area, the samples were sampled and remodeled at the site. Extensive experimental tests were conducted to investigate the relationship between the hygroscopic expansion rate and the water content of the expansive soil under different initial moisture content, dry density, and free load. The results showed that, under the condition of natural initial water content and dry density, although the hygroscopic expansion rate of the medium expansive soil was nonlinear with the subsequent water content, in the range of large water content (within about 50\%), the expansive soil swelled linearly. There was a linear relationship between the rate and the water content. With the increase of the initial water content, the hygroscopic expansion rate and expansion rate of the expansive soil decreased. With the increase of the dry density, the hygroscopic expansion rate and the expansion rate of the expansive soil increased. The water absorption performance did not decrease, and the soil continued to maintain the previous moisture absorption rate and expansion rate after the soil reached saturation, while after the water content reached 1.5 2.0 times the saturated water content, the soil moisture absorption expansion rate gradually decreased until it finally stabilized. The slope $k$ of the expansion rate increased with the initial dry density and decreased with the initial moisture content. As dry density was increased, the slope $k$ was increased at an increased rate. Moreover, as the initial moisture content was decreased, the slope $k$ was increased at an increased rate.

\section{Introduction}

Expansive soil has the characteristics of expansion and shrinkage of water sucking and water losing, which are two of the most essential characteristics of expansive soil. The causes and mechanisms of the expansion and shrinkage deformation of expansive soil depend not only on the material composition of the expansive soil, but also on the spatial structural characteristics of these constituents in the soil. The content of hydrophilic mineral components and the spatial characteristics of basic microstructure units are the internal factors that determine the expansion and shrinkage characteristics of expansive soils. Under the external factors (overburden pressure, rainwater action, and compaction), the expansion and shrinkage characteristics of expansive soils will also be different.
Recently, many studies have been conducted to investigate deformation characteristic of expansive soil. For example, Holtz and Gibbs [1] studied the swelling behavior of expansive soil under different initial water content, dry density, and overburden load conditions. A good relationship between swelling deformation and water content of expansive soil is obtained. Under the same overburden pressure, several studies found that expansion deformation of expansive soil varies linearly with the initial water content [2-5]. At a given initial water content, $\mathrm{Xu}$ and Lei [6] found that the expansion of Shanxi expansive soil has a negative exponential relationship with the overburden pressure. Several other studies explore the effects of compaction degree and the initial dry density on the expansibility of compacted expansive soil $[7,8]$. The relationships between the same initial water content, the expansion ratio, and 
compaction of expansive soil are proposed. Under the same dry density condition, the expansive soil expansion deformation has a linear relationship with the initial water content.

In seasonal frozen regions, expansive soils inevitably suffer freezing-thawing cycles [9]. It is found that a moderate freezing temperature has the biggest effects on the volumetric changes of expansive soils. Moreover, several studies investigate the effects of temperature on the behavior of collapse loess $[10,11]$. When expansive soils are subjected to water changes, excessive deformation may be induced, which may threaten underground structures [12-17]. Since expansion and shrinkage are induced in expansive soils when subjected to water changes, more and more studies have been conducted to the deformation characteristic of reinforced expansive soil [18]. By conducting an extensive laboratory tests, strength characteristics of expansive soil treated by jute fiber reinforcement are investigated. The contribution of jute fibers on enhancing strength parameters is identified [19]. Based on experimental tests, Lu et al. [20] explored freeze-thaw performance of cement-treated expansive soils. It is found that cement-treated expansive soils become less sensitive to moisture.

The current researches show that, under the influence of a single factor, the relationship between the expansion rate and water content of expansive soil is mostly linear, but the change of water content is mostly less than $40 \%$. In order to find the change law of swelling rate of medium expansive soil with the water content, especially when the soil reaches saturation, even after the soil is saturated, in this paper, the swelling soil in Hefei was taken for remodeling, and the relationship between its swelling rate and initial moisture content, subsequent moisture content, and dry density was studied. The water content at the end of the test was about $60 \%$.

\section{Test Methods}

The humidification expansion rate of expansive soil is also affected by other factors, such as load conditions and moisture absorption methods. This test ignores the influence of load and water injection methods and studies the relationship between the expansion ratio of expansive soil and water content $t$ under free load, confined, and infiltration conditions, when the soil has different initial water content and different dry density.

According to the investigation data, the natural water content of expansive soil in the deep cut section of water diversion from Yangtze River to Huaihe River is $15 \%-30 \%$, and the dry density of soil is $1.4-1.65 \mathrm{~g} / \mathrm{cm}^{3}$. In the test, the initial moisture content of soil mass is taken as $15 \%$ 20\%, and the dry density of soil mass is controlled as $1.45 \sim 1.6 \mathrm{~g}$ $/ \mathrm{cm}^{3}$, which is basically consistent with the actual situation on site. The same batch of typical medium undisturbed expansive soil (free expansion rate of 70\%) was reshaped to control its initial moisture content and dry density. The test consisted of seven groups, as shown in Table 1.

The test methods are as follows: firstly, the standard soil samples with different initial conditions are prepared by ring
TABLE 1: Initial condition control of expansive soil.

\begin{tabular}{lcc}
\hline Numbering & Initial moisture content $(\%)$ & Dry density $\left(\mathrm{g} / \mathrm{cm}^{3}\right)$ \\
\hline 1 & 15.0 & 1.45 \\
2 & 15.0 & 1.53 \\
3 & 17.5 & 1.45 \\
4 & 17.5 & 1.53 \\
5 & 20.0 & 1.45 \\
6 & 20.0 & 1.53 \\
7 & 20.0 & 1.60 \\
\hline
\end{tabular}

knife method; secondly, the soil samples are soaked in water (each sample has different amount of immersion); thirdly, the height change of soil samples after immersion is measured; finally, the soil samples are dried to calculate the moisture content change of each soil sample.

\section{Experiment Process}

(1) 24 expansive soil samples with the same initial conditions (initial moisture content and dry density) in each group were created.

(2) The soil samples were then immersed in water with confined and free load conditions, and the moisture content of each soil sample was different.

(3) Immersing the soil samples and measuring the height change of the soil samples with a dial indicator for a period of time (controlled by $24 \mathrm{~h}$, Cheng et al. [10] and Meng et al. [12] showed that, under the condition of no load, the moisture absorption of the expansive soil gradually stabilized after $6 \mathrm{~h}$ and after 24 hours, and expansion was no longer changed).

(4) The soil sample was put into the drying box for moisture content determination;

(5) After 24 hours, the dried soil samples were taken out, and the weight of each soil sample was measured.

(6) The moisture content of each soil sample was calculated.

(7) A curve of the relationship between the expansion ratio and the water content of the soil samples (the initial conditions were the same) was drawn. The test process is shown in Figure 1.

\section{Test Results}

4.1. Relationship between Expansion Ratio and Initial Moisture Content and Dry Density. The soil was firstly prepared according to the dry density. At the initial moisture content, the soil sample expansion rate was assumed to be 0 , and then the same batch of soil samples was added with different water contents. After 24 hours, the moisture content and the free load expansion ratio were measured. Under different initial conditions, the relationship between the free load expansion ratio and the water content is shown in Figures 2 and 3. It can be seen from the figures that, in the early stage (subsequent moisture content is less than $w_{1}$ ), under different initial conditions, the relationship between the free load expansion ratio and the water content is approximately 


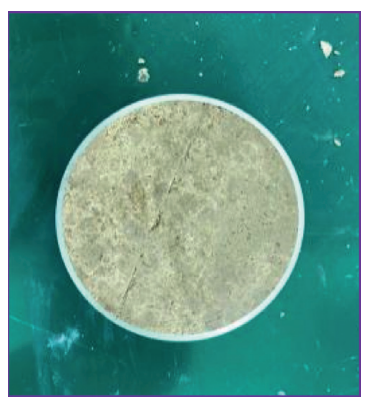

(a)

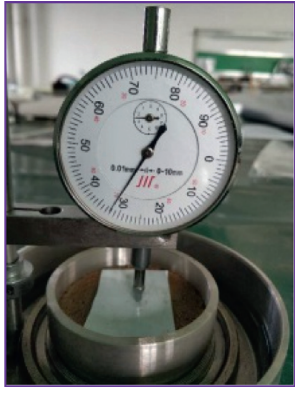

(b)

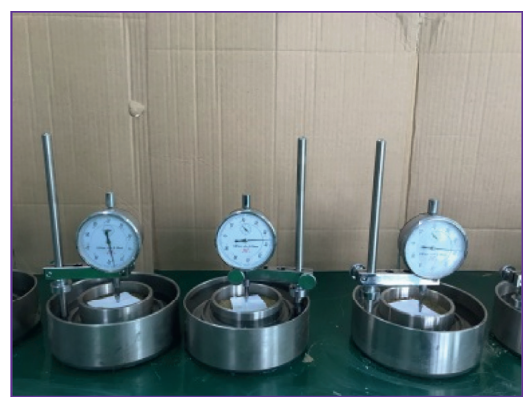

(c)

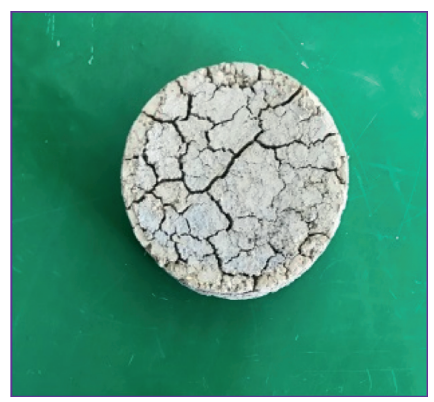

(d)

Figure 1: Test process. (a) Sample, (b) loading, (c) immersion, and (d) drying.
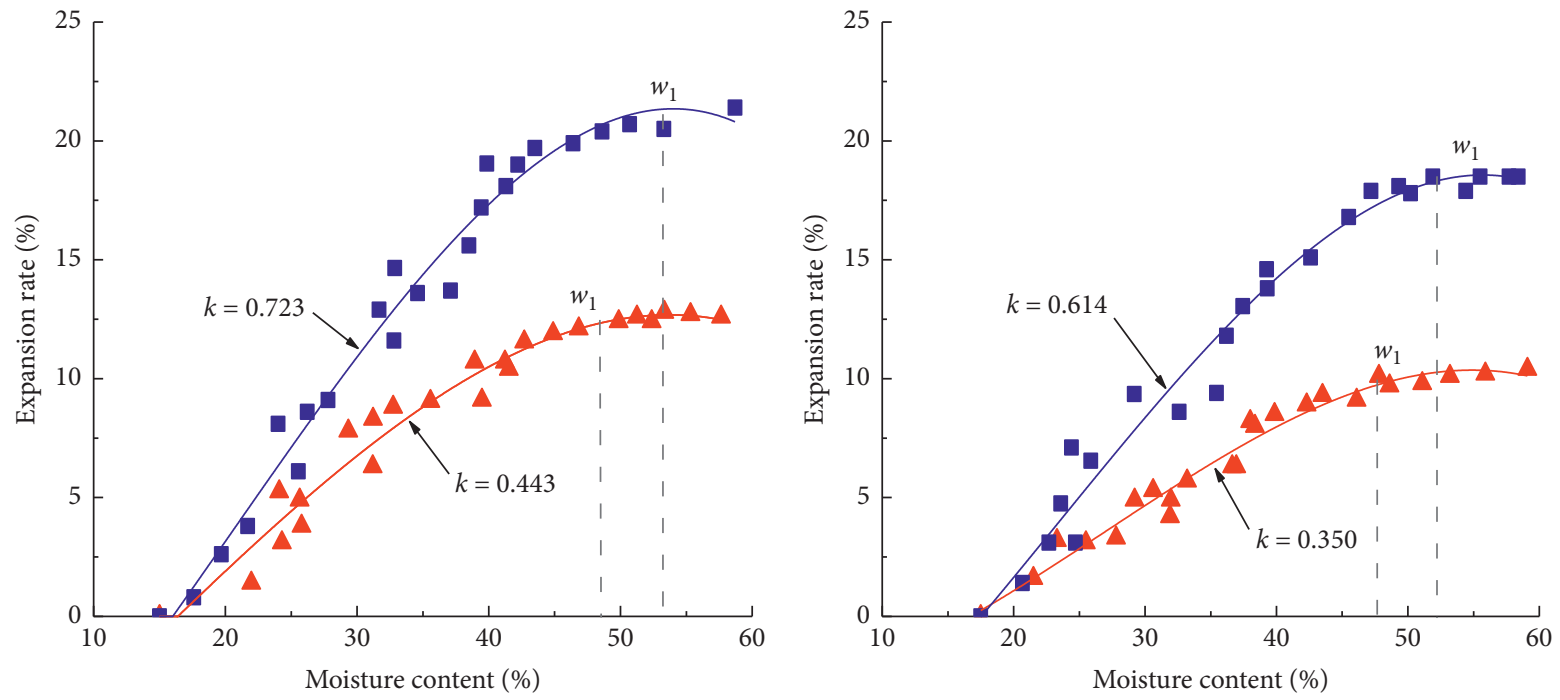

$\Delta$ - dry density $1.45 \mathrm{~g} / \mathrm{cm}^{3}$

- dry density $1.53 \mathrm{~g} / \mathrm{cm}^{3}$

$\Delta-$ dry density $1.45 \mathrm{~g} / \mathrm{cm}^{3}$

- - dry density $1.53 \mathrm{~g} / \mathrm{cm}^{3}$

(a)

(b)

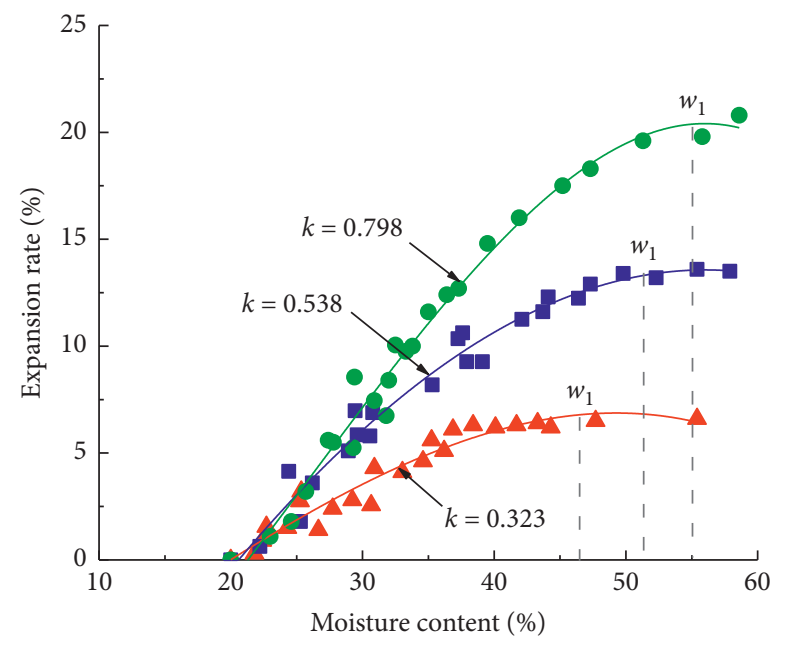

$\Delta$ - dry density $1.45 \mathrm{~g} / \mathrm{cm}^{3}$
- dry density $1.53 \mathrm{~g} / \mathrm{cm}^{3}$
- dry density $1.45 \mathrm{~g} / \mathrm{cm}^{3}$

(c)

Figure 2: The relationship between the free load swelling rate and the initial moisture content. (a) Initial moisture content 15\%, (b) initial moisture content $17.5 \%$, and (c) initial moisture content $20 \%$. 


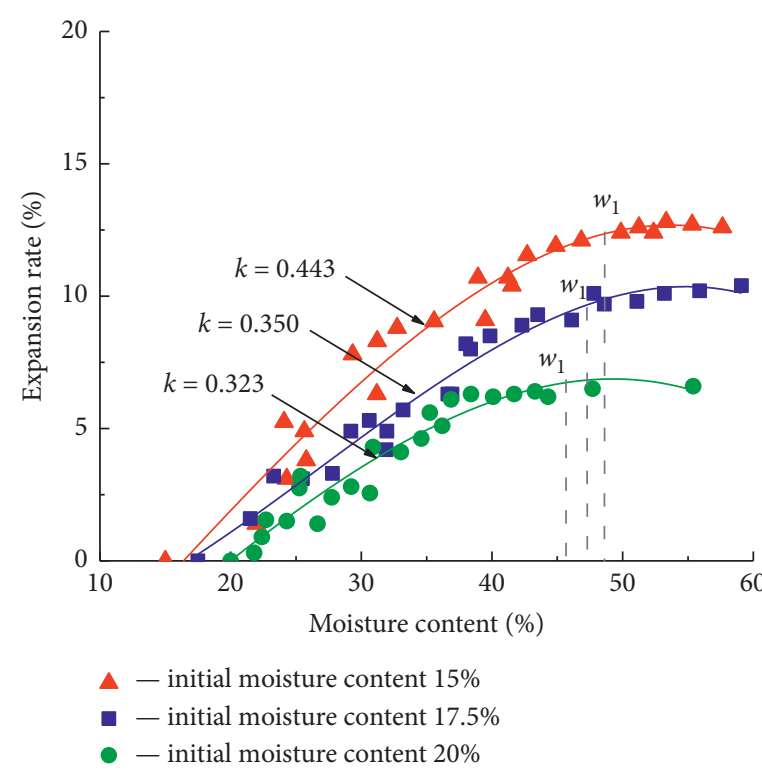

(a)

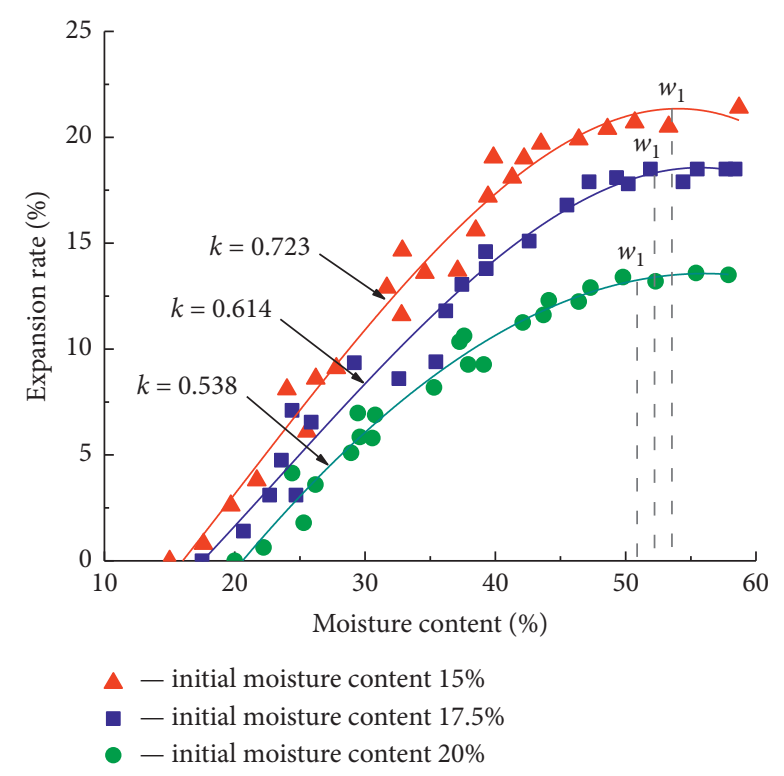

(b)

Figure 3: The relationship between the free load swelling rate and dry density. (a) Dry density $1.45 \mathrm{~g} / \mathrm{cm}^{3}$ and (b) dry density $1.53 \mathrm{~g} / \mathrm{cm}^{3}$.

linear (the slope of the straight line is $k$, where the water content is less than 40\%). Under the same subsequent moisture content, the soil expansion rate decreases with the increase of the initial water content, while as the dry density increases, the soil expansion rate increases. Under the same initial water content and the same dry density, the growth rate of soil expansion rate is also larger in the early stage. In the later stage (subsequent water content is greater than $w_{1}$ ), as the water content increases, the growth rate of soil expansion rate decreases significantly, gradually becoming steady. Under the same initial water content, the soil moisture absorption expansion rate increases with the increase in the dry density. Under the same dry density condition, the soil moisture absorption expansion rate decreases with the increase of initial water content.

In fact, when the water content is within a certain range, the expansion ratio and water content of the expansive soil can be regarded as a piecewise linear relationship. When the moisture content of the expansive soil is extremely low (below 10\%), the slope of the linear fitting relationship between the expansion ratio and the water content is larger [7], while when the water content is higher (after saturation), the slope of the linear fitting relationship between the expansion ratio and the water content becomes gradually smaller. This test is based on the fact that the expansive soil (natural moisture content is $15 \%$ to $30 \%$ ) is affected by rainfall, and the relationship between the expansion rate and the water content is basically linear under the condition of normal change of water content (increased or close to water content). When the water content increases to a certain extent, the soil moisture absorption expansion is weakened, and the soil gradually reaches the limit of hygroscopic expansion under the corresponding initial conditions. Rao et al.'s [7] research on the swelling of expansive soils after water sucking showed that the expansive soil has the highest hygroscopic expansion rate when the soil moisture content is less than 10\%. From 10\% to $35 \%$, the expansive soil moisture absorption expansion rate decreases, and when the water content is greater than $35 \%$, at the time, the soil is nearly saturated or saturated, and its expansion rate is greatly reduced. The relationship between the expansion ratio of expansive soil and water content is close to piecewise linearity: less than $10 \%, 10 \%$ to $35 \%$, and more than $35 \%$.

4.2. Relationship between the Slope of the Linear Segment and the Initial Conditions. When the moisture content of expansive soil is less than $40 \%$, the relationship between the free load swelling rate and initial moisture content is close to linear. The relationship between the slope $k$ and the initial conditions is shown in Figure 4. As can be seen in the figure, the slope increases with the initial dry density and decreases with the initial moisture content. As dry density increases, the slope $k$ accelerates increasing, and as the initial moisture content decreases, the slope $k$ accelerates increasing too. Though when the initial moisture content is low, and the dry density is high, the slope $k$ changes dramatically. The changes of the slope $k$ of different initial moisture content with dry density are close to parallel state, and the changes of the slope $k$ of different dry density with initial moisture content are close to parallel state too. This suggests that the influence of the single condition to the slope $k$ only depends on the state of the other initial conditions.

Supposing that the change in the slope $k$ is $k^{\prime}$ (the slope of $k$ ), the average value of $k^{\prime}$ is 3.30 in the relationship to the dry density, and the average value of $k^{\prime}$ is 3.05 in the relationship to the initial moisture content. The change range of $k$ value caused by the change of dry density at the same initial moisture content is slightly larger than that caused by 


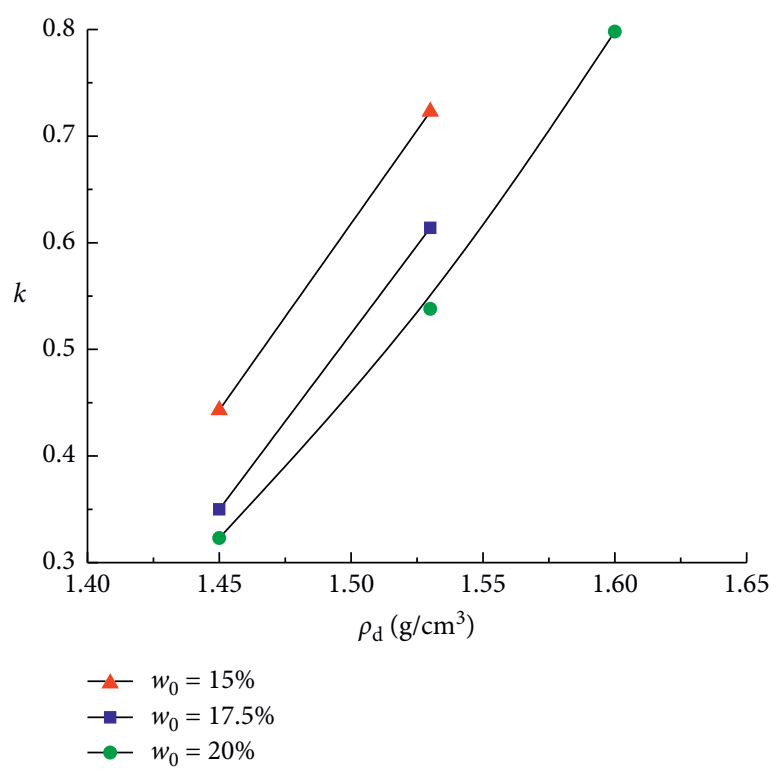

(a)

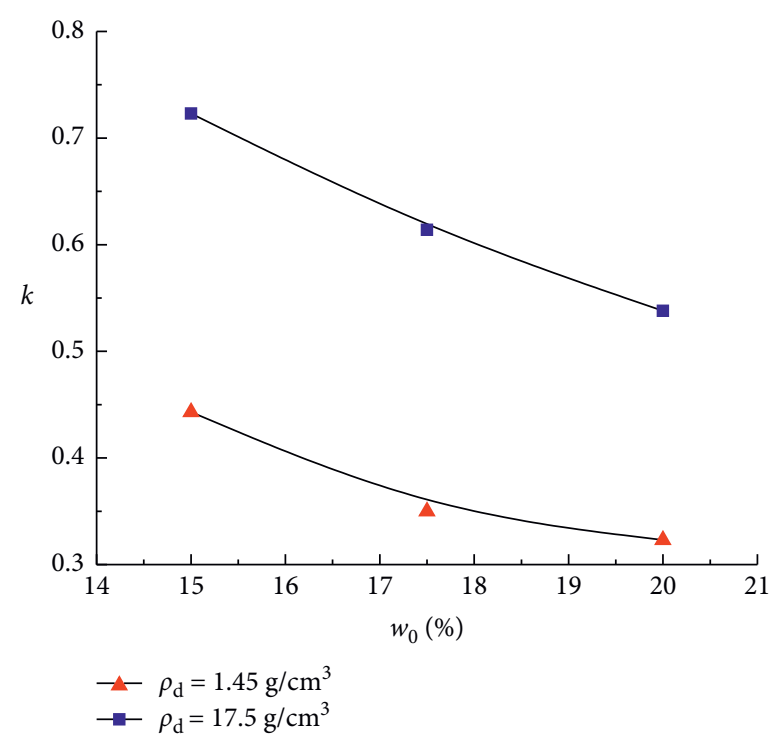

(b)

Figure 4: The relationship between the slope of the linear segment and the initial conditions. (a) Relationship to dry density and (b) relationship to initial the moisture content.

the change of initial moisture content at the same dry density. It shows that the effect of dry density on the free load expansion rate of medium expansive soil is greater than that of the initial moisture content.

\subsection{Relationship between Moisture Content of Inflection Point} and Saturated Water Content. According to the test results, when the water content is less than $40 \%$, the relationship between the expansion ratio of the expansive soil and the water content is approximately linear and can be expressed by the following formula:

$$
\delta_{\mathrm{e}}=a w+b .
$$

In the formula, $a, b$ are fitting parameters; $\delta_{\mathrm{e}}=\left(\left(H_{\mathrm{f}}-H_{\mathrm{i}}\right) / H_{0}\right) \times 100 \%, H_{\mathrm{f}}$ is the dial gauge reading $(\mathrm{mm})$ after hygroscopic stabilization; $H_{\mathrm{i}}$ is the dial gauge reading $(\mathrm{mm})$ at the beginning of the test; $H_{0}$ is the initial height of the sample. The expansive soil particles have a specific gravity of $G_{s}$, a dry density of soil $\rho_{\mathrm{d}}$, and an initial void ratio $e_{0}$ of:

$$
e_{0}=\frac{G_{\mathrm{s}}}{\rho_{\mathrm{d}}}-1
$$

In the formula, $G_{s}=2.61$.

It is assumed that the initial soil moisture content is $w_{0}$, the subsequent water content is $w$, and the soil density $\rho$ after expansion can be expressed as

$$
\rho=\frac{G_{\mathrm{s}} * 1 *(1+w)}{\left(1+e_{0}\right)\left(1+\delta_{\mathrm{e}}\right)} .
$$

Assuming that the soil void ratio after expansion is assumed to be $e$, the soil density can be expressed as

$$
\rho=\frac{G_{\mathrm{s}} * 1 *(1+w)}{1+e} .
$$

The void ratio of the soil after expansion is

$$
e=\left(1+e_{0}\right)\left(1+\delta_{\mathrm{e}}\right)-1 .
$$

Suppose that the saturated water content of the soil is less than $40 \%$, and the expansion rate of the saturated soil is

$$
\delta_{\text {esat }}=a w_{\text {sat }}+b .
$$

The saturated water content of the soil can be expressed as

$$
w_{\text {sat }}=\frac{m_{\mathrm{wsat}}}{m_{\mathrm{s}}}=\frac{\left[\left(1+e_{0}\right)\left(1+\delta_{\text {esat }}\right)-1\right] \rho_{\mathrm{w}}}{\left(1+e_{0}\right) \rho_{\mathrm{d}}} .
$$

Combine formula (6):

$$
w_{\text {sat }}=\frac{\rho_{\mathrm{w}}\left[\left(1+e_{0}\right)(1+b)-1\right]}{\left(1+e_{0}\right)\left(\rho_{\mathrm{d}}-a \rho_{\mathrm{w}}\right)} .
$$

As can be seen in Table 2, the saturated soil moisture content of the expansive soil used in the test is less than $40 \%$, and the water content is in the linear phase of the expansion ratio-water content curve. The water content of the inflection point, where the water absorption of the expansive soil tends to be stable, is greater than the saturated water content. This indicates that the expansive soil can continue to absorb water quickly after saturation, and the initial moisture content of the soil is low, the dry density is large, and the water absorption capacity is strong. The water content of the expansive soil moisture inflection point is about $50 \%$. Under different initial conditions, the moisture content of the moisture inflection point is not much different. The ratio of the moisture content of the inflection point to the saturated 
TABLE 2: Saturated soil moisture content of expansive soil under different initial conditions.

\begin{tabular}{|c|c|c|c|c|c|}
\hline $\begin{array}{l}\text { Initial moisture content } \\
(\%)\end{array}$ & $\begin{array}{l}\text { Dry density } \\
\left(\mathrm{g} / \mathrm{cm}^{3}\right)\end{array}$ & $\begin{array}{l}\text { Initial void } \\
\text { ratio }\end{array}$ & $\begin{array}{l}\text { Saturated moisture content } w_{\text {sat }} \\
(\%)\end{array}$ & $\begin{array}{l}\text { Inflection point moisture content } w_{1} \\
\qquad(\%)\end{array}$ & $w_{1} / w_{\text {sat }}$ \\
\hline 15.0 & 1.45 & 0.80 & 37 & 48 & 1.28 \\
\hline 15.0 & 1.53 & 0.71 & 38 & 53 & 1.41 \\
\hline 17.5 & 1.45 & 0.80 & 35 & 47 & 1.34 \\
\hline 17.5 & 1.53 & 0.71 & 34 & 52 & 1.53 \\
\hline 20.0 & 1.45 & 0.80 & 34 & 46 & 1.36 \\
\hline 20.0 & 1.53 & 0.71 & 31 & 51 & 1.63 \\
\hline 20.0 & 1.60 & 0.63 & 27 & 55 & 2.02 \\
\hline
\end{tabular}

water content is between 1.3 and 2.0, and the initial moisture content is low, the dry density is large, and the ratio is also large.

\section{Conclusion}

Through the study of the relationship between the free load expansion ratio of the expansive soil and the initial water content and dry density, and the relationship between the moisture content of the inflection point of the expansive soil and the saturated water content, the following beneficial conclusions are obtained:

(1) Under the condition of natural initial water content and dry density, although there is a nonlinear relationship between the moisture absorption expansion ratio of the medium expansive soil and the subsequent moisture content, in the range of large water content (within about 50\%), the expansion ratio of expansive soil has an approximate linear relationship with water content.

(2) With the increase of initial water content, the hygroscopic expansion ratio and expansion rate of expansive soil decrease, and with the increase of dry density, the hygroscopic expansion ratio and expansion rate of expansive soil increase.

(3) In the linear stage, the slope $k$ increases with the initial dry density and decreases with the initial moisture content. As dry density is increased, the slope $k$ increased at an increased rate. Moreover, as the initial moisture content is decreased, the slope $k$ is also increased at an increased rate.

(4) After the hygroscopicity of the expansive soil is saturated, its water absorption performance will not decrease, and the soil will continue to maintain the previous moisture absorption rate and expansion rate. After the water content reaches 1.5 to 2.0 times the saturated water content, the soil moisture absorption expansion rate will gradually decrease until it finally stabilizes.

\section{Data Availability}

In this study, all the data are obtained by conducting laboratory tests. All the data used to support the findings of this study are included within the article.

\section{Conflicts of Interest}

The authors declare that they have no known conflicts of interest or personal relationships that could have appeared to influence the work reported in this paper.

\section{Acknowledgments}

This work was supported by the Fundamental Research Funds for the Central Public Welfare Research Institutes (No. tks190203).

\section{References}

[1] W. G. Holtz and H. J. Gibbs, "Engineering properties of expansive clays," Theoretical Biology \& Medical Modelling, vol. 121, no. 1, pp. 269-276, 1956.

[2] Y. F. Xu, J. Q. Dai, and Z. Z. Yin, "Preliminary study on the model of swelling deformation of some expansive soil in Ningxia," Journary of Basic Science and Engineering, vol. 5, no. 2, pp. 161-166, 1997.

[3] Y. F. Xu, Z. G. Wu, and C. X. Liu, "Relativity between compaction conditions and swelling deformation of expansive soils," Journal of Hohai University, vol. 25, no. 3, pp. 57-60, 1997.

[4] J. Q. Li, Q. C. Wang, and R. L. Zhang, "Experimental study on swelling law of expansive soil during humidification," HydroScience and Engineering, vol. 3, pp. 86-94, 2018.

[5] J. Q. Li, Q. C. Wang, and R. L. Zhang, "Influence of water injection mode on swelling amount of expansive soil," Water Resources and Hydropower Engineering, vol. 49, no. 6, pp. 179-184, 2018.

[6] Y. Xu and S. Y. Lei, "Analysis of variance affecting linear swelling ratio," Journal of Northwestern Institute of Architectutal Engineering (Natural Sciences), vol. 20, no. 2, pp. 6-9, 2003.

[7] X. B. Rao, B. Huang, and Y. G. Wu, "Experimental study of expansion characteristics of compacted expansive soil," Engineering Journal of Wuhan University, vol. 44, no. 2, pp. 211-215, 2011.

[8] M. V. Villar and A. Lloret, "Influence of dry density and water content on the swelling of a compacted bentonite," Applied Clay Science, vol. 39, no. 1-2, pp. 38-49, 2008.

[9] Y. Lu, S. Liu, E. Alonso, L. Wang, L. Xu, and Z. Li, "Volume changes and mechanical degradation of a compacted expansive soil under freeze-thaw cycles," Cold Regions Science and Technology, vol. 157, pp. 206-214, 2019.

[10] Q. Cheng, C. Zhou, C. W. W. Ng, and C. Tang, "Thermal effects on water retention behaviour of unsaturated collapsible 
loess," Journal of Soils and Sediments, vol. 20, no. 2, pp. 756-762, 2020.

[11] C. W. W. Ng, Q. Cheng, and C. Zhou, "Thermal effects on yielding and wetting-induced collapse of recompacted and intact loess," Canadian Geotechnical Journal, vol. 55, no. 8, pp. 1095-1103, 2018.

[12] K. Meng, C. Cui, Z. Liang, H. Li, and H. Pei, "A new approach for longitudinal vibration of a large-diameter floating pipe pile in visco-elastic soil considering the three-dimensional wave effects," Computers and Geotechnics, vol. 128, p. 103840, 2020.

[13] C. Cui, K. Meng, C. Xu, Z. Liang, H. Li, and H. Pei, "Analytical solution for longitudinal vibration of a floating pile in saturated porous media based on a fictitious saturated soil pile model," Computers and Geotechnics, vol. 131, p. 103942, 2021.

[14] Y. X. Wang, S. B. Shan, C. Zhang, and P. P. Guo, "Seismic response of tunnel lining structure in a thick expansive soil stratum," Tunnelling and Underground Space Technology, vol. 88, pp. 250-259, 2019.

[15] J. Shi, C. Ding, C. W. W. Ng, H. Lu, and L. Chen, "Effects of overconsolidation ratio on tunnel responses due to overlying basement excavation in clay," Tunnelling and Underground Space Technology, vol. 97, p. 103247, 2020.

[16] J. Shi, J. Wei, C. W. W. Ng, and H. Lu, "Stress transfer mechanisms and settlement of a floating pile due to adjacent multi-propped deep excavation in dry sand," Computers and Geotechnics, vol. 116, p. 103216, 2019.

[17] J. W. Shi, Y. Wang, and Y. H. Chen, "A simplified method to estimate curvatures of continuous pipelines induced by normal fault movement," Canadian Geotechnical Journal, vol. 55, pp. 343-352, 2018.

[18] S. Bhuvaneshwari, R. G. Robinson, and S. R. Gandhi, "Resilient modulus of lime treated expansive soil," Geotechnical and Geological Engineering, vol. 37, no. 1, pp. 305-315, 2019.

[19] Y. X. Wang, P. P. Guo, W. X. Ren, B. X. Yuan, and H. P. Yuan, "Laboratory investigation on strength characteristics of expansive soil treated with jute fiber reinforcement," International Journal of Geomechanics, vol. 17, no. 11, p. 04017101, 2017.

[20] Y. Lu, S. Liu, Y. Zhang, Z. Li, and L. Xu, "Freeze-thaw performance of a cement-treated expansive soil," Cold Regions Science and Technology, vol. 170, p. 102926, 2019. 